\title{
Needle track seeding in hepatocellular carcinoma after local ablation by high-dose-rate brachytherapy: a retrospective study of 588 catheter placements
}

\author{
Robert Damm, MD!', Ingo Zörkler, MD', Bela Rogits, MD², Peter Hass, MD³, Jazan Omari, MD', Maciej Powerski, MD, PhD', \\ Prof. Sigrfried Kropf ${ }^{4}$, Konrad Mohnike, MD ${ }^{5}$, Prof. Maciej Pech, MD', Prof. Jens Ricke, MD' , Prof. Max Seidensticker, MD 6 \\ 'Department of Radiology and Nuclear Medicine, Otto-von-Guericke University, Magdeburg, 2Pawlow Poliklinik, Radiologische \\ Gemeinschaftspraxis, Magdeburg, ${ }^{3}$ Department for Radiation Oncology. Otto-von-Guericke University, Magdeburg, ${ }^{4}$ Institute for Biometry \\ and Medical Informatics, Otto-von-Guericke University, Magdeburg, ${ }^{5}$ Diagnostic and Therapeutic Center am Frankfurter Tor, Berlin, \\ ${ }^{\circ}$ Department of Radiology, Ludwig-Maximilians-University, Munich, Germany
}

\begin{abstract}
Purpose: Needle track seeding in the local treatment of hepatocellular carcinoma (HCC) is not yet evaluated for catheter-based high-dose-rate brachytherapy (HDR-BT), a novel local ablative technique.

Material and methods: We report a retrospective analysis of 100 patients treated on 233 HCC lesions by HDR-BT (using 588 catheters in total). No needle or catheter track irradiation was used. Minimum required follow-up with imaging was 6 months. In case of suspected needle track seeding (intra- and/or extrahepatic) in follow-up, image fusion of follow-up CT/MRI with 3D irradiation plan was used to verify the location of a new tumor deposit within the path of a brachytherapy catheter at the time of treatment.

Results: We identified 9 needle track metastases, corresponding to a catheter-based risk of $1.5 \%$ for any location of occurrence. A total of 7 metastases were located within the liver (catheter-based risk, 1.2\%), and 2 metastases were located extrahepatic (catheter-based risk, $0.3 \%$ ). Eight out of 9 needle track metastases were successfully treated by further HDR-BT.

Conclusions: The risk for needle track seeding after interstitial HDR-BT of HCC is comparable to previous reports of percutaneous biopsies and radiofrequency ablation (RFA), especially in case of extrahepatic needle track metastases. To compensate for the risk of seeding, a track irradiation technique similar to track ablation in RFA should be implemented in clinical routine.

J Contemp Brachytherapy 2018; 10, 6: 516-521 DOI: https://doi.org/10.5114/jcb.2018.80626
\end{abstract}

Key words: hepatocellular carcinoma, local ablative treatment, needle track seeding.

\section{Purpose}

Hepatocellular carcinoma (HCC) is a primary liver tumor most often found in patients with liver cirrhosis and/ or viral hepatitis. Its incidence has increased over the last years worldwide [1]. Beneath surgical resection, local ablative (e.g., radiofrequency ablation - RFA, microwave ablation - MWA) and loco-regional (e.g., transarterial chemoembolization - TACE) treatments are favored for early to intermediate stage of HCC. However, these treatments may not be suitable for every patient due to technical restrictions $[2,3,4]$. Thermal ablation techniques have their limitations, especially in location close to vulnerable structures (e.g., bile ducts) and lesion size of 3.5 to $4 \mathrm{~cm}$, while loco-regional techniques require sufficient, vascular access for the application of embolic agents, showing lack in local control if tumor nodules exceed a size of 5 to $7 \mathrm{~cm}[5,6]$. Thus, computed tomography (CT)-guided high-dose-rate brachytherapy (HDR-BT) as a form of catheter-based radiotherapy is a promising treatment option for tumors not accessible for thermal ablation techniques as well as an alternative to TACE. By inserting an Iridium 192 source through percutaneously applied catheters, interstitial brachytherapy has no technical restrictions in lesion size to deliver potentially ablative doses, and can be employed close to central structures $[7,8]$. In a series of studies, the safety and effectiveness of HDR-BT has already been 
demonstrated, suggesting a potential as a bridging therapy to liver transplantation in addition to radiofrequency ablation or transarterial chemoembolization [9].

The risk of spreading malignant cells during diagnostic and therapeutic methods have been reported for liver biopsy and thermal ablation with heterogeneous results, also varying by the utility of needle track ablation $[10,11,12,13]$. As the catheter placement for HDR-BT comprises an initial puncture (including possible corrections of the needle position) and insertion of catheter sheets in Seldinger's technique, a corresponding risk of dislocating tumor cells through manipulation should be assumed.

The risk of needle track seeding after HDR brachytherapy, particularly in case of the potential utility as a bridging treatment for liver transplantation in early stage HCC, should be further investigated. On the other side, patients with larger tumor volumes in intermediate stage of the disease might have an increased risk for needle track seeding, as more catheter placements are required for a sufficient dose distribution [14]. In this retrospective study, we report needle track seeding after HDR brachytherapy in a series of 100 patients, with a total of 588 catheter placements for local ablation of $233 \mathrm{HCC}$ lesions. No catheter or needle track irradiation had been used in these patients.

\section{Material and methods}

\section{Eligibility criteria and patient cohort}

We retrospectively analyzed patients who underwent interstitial HDR brachytherapy for HCC between 2006 and 2012. All lesions were previously proven either by core needle biopsy or by matching the non-invasive criteria of HCC in CT or magnetic resonance imaging (MRI) [15], according to the Clinical Practice Guidelines of the European Association for the Study of the Liver (EASL) released in 2012 [16].

100 patients (83 males, 17 females), with 233 HCC nodules and a total of 588 catheter placements met the inclusion criteria (see section follow-up). The average age at the time of intervention was $68 \pm 8.1$ years ( $44-82$ years). Nearly all patients had an underlying liver cirrhosis $(n=98)$, mainly caused by alcohol consumption $(n=28)$ or viral hepatitis $(n=22)$. Infrequent causes of cirrhosis were non-alcoholic steatohepatitis $(n=8)$ and hemochromatosis $(n=2)$. In all other cases, the etiology of cirrhosis was cryptogenic $(n=38)$.

Only a minority of patients presented with extrahepatic disease including lymphatic $(n=5)$ or distant metastases $(n=5)$. A summary of patient and treatment characteristics is presented in Table 1.

Table 1. Patient and treatment characteristics and analysis on influencing factors for needle track seeding

\begin{tabular}{|c|c|c|c|c|}
\hline Variable & $\%(N)$ or mean \pm SD & Patient-based $p$ & Lesion-based $p$ & Catheter-based $p$ \\
\hline Male/female & $83 \%(83) / 17 \%(17)$ & 0.66 & 0.49 & 0.33 \\
\hline Age (years) & $68.0 \pm 8.1$ & 0.26 & 0.21 & 0.37 \\
\hline Liver cirrhosis & $98 \%(98 / 100)$ & & & \\
\hline hemochromatosis & $2 \%(2 / 98)$ & 1.0 & & \\
\hline viral hepatitis & $22 \%(22 / 98)$ & 1.0 & & \\
\hline ASH & $29 \%(28 / 98)$ & 0.99 & & \\
\hline NASH & $8 \%(8 / 98)$ & 0.99 & & \\
\hline other & $39 \%(38 / 98)$ & & & \\
\hline HCC grading & $62 \%(62 / 100)$ & 0.54 & 0.23 & 0.3 \\
\hline well & $39 \%(24 / 62)$ & & & \\
\hline moderate & $55 \%(34 / 62)$ & & & \\
\hline poor & $6 \%(4 / 62)$ & & & \\
\hline Concomitant sorafenib treatment & $22 \%(22 / 78)$ & 0.6 & 0.96 & 0.62 \\
\hline Pseudo-capsular HCC & $8 \%(18 / 233)$ & 0.98 & & \\
\hline Lesion size $(\mathrm{cm})$ & $3.3 \pm 2.6$ & 0.2 & 0.78 & 0.09 \\
\hline Ablation dose (Gy) & $16.5 \pm 11.6$ & 0.65 & 0.7 & 0.59 \\
\hline Overpenetration (per catheter) & $9 \%(53 / 588)$ & 0.23 & 0.69 & \\
\hline \multicolumn{5}{|l|}{ Catheter insertion lengths (cm) } \\
\hline /patient & $74.8 \pm 57.4$ & 0.94 & & \\
\hline /lesion & $32.1 \pm 37.4$ & & 0.78 & \\
\hline /catheter & $12.7 \pm 31.2$ & & & 0.75 \\
\hline
\end{tabular}



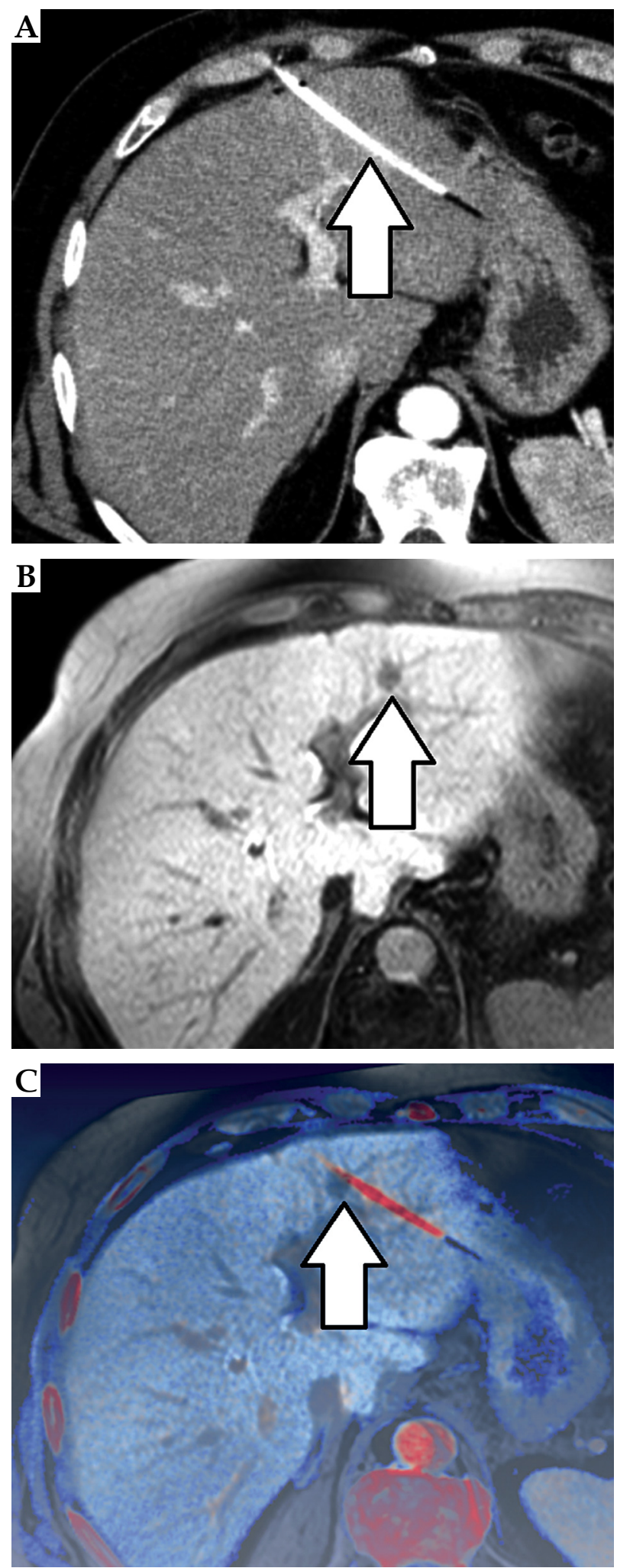

Fig. 1. Image fusion data set: peri-interventional CT showing HDR brachytherapy catheter (arrow, A), follow-up MRI suspecting a needle track lesion (arrow, B), axial image fusion of follow-up MRI and planning CT of HDR brachytherapy confirming the origin of the new lesion within the former path of the brachytherapy catheter (arrow, C)

\section{HDR brachytherapy technique}

In order to place an Iridium 192 source directly in the HCC lesions, irradiation catheters must be inserted into the tumor. The access for a soft catheter is accomplished by a percutaneous puncture with an 18 Ga coaxial needle under image guidance (CT or open MRI fluoroscopy) and subsequent insertion of an angiographic catheter sheet in Seldinger's technique. The irradiation catheter is then placed inside the catheter sheath and fixed by a single suture. For planning purposes, diagnostic imaging (e.g., contrast enhanced CT) is performed after complete catheter placement. Afterwards, a three-dimensional treatment plan is generated based on diagnostic imaging data acquired following catheter placement. Generally, the preferred surrounding dose for HCC is 15 Gy. After successful delivery of the desired dose in a single fraction, the catheters and sheaths are removed leaving absorbable gelatin sponge in the track. Concomitant conscious sedation is achieved by individual administration of fentanyl and midazolam. A further description of irradiation technique and concomitant treatment is presented elsewhere [17].

\section{Follow-up}

All eligible patients required a follow-up consisting of CT or MRI at least 3 and 6 months after therapy, with a dynamic contrast-enhanced scan protocol including arterial, portal venous, and late venous phase. Any new intrahepatic lesion with a diameter of at least $1 \mathrm{~cm}$ and arterial enhancement with venous wash out was defined as an intrahepatic recurrence of HCC, while clear tumor growth outside the liver was sufficient for the definition of extrahepatic lesions [16].

Subsequent therapies in the follow-up period included sorafenib $(n=22)$, transarterial chemoembolization $(n=18)$, Y90 radioembolization $(n=4)$, and radiofrequency ablation $(n=4)$.

\section{Imaging analysis}

We determined the primary tumor size, number and location of metastases, the total length of each catheter from the skin to the tip as well as 'over-penetration' of the tip beyond the HCC lesion.

As a first step, the available image data sets were evaluated for the probability of needle tract seeding according to the following definitions: 1) Temporal causality: needle track seeding should be diagnosed after therapy within a reasonable timeframe of 2 years; 2) Local causality: needle tract seeding had to be situated around a prior catheter track within a margin of $1 \mathrm{~cm}$.

In a second step, the suspected needle track metastases had to be objectively verified. Amira ${ }^{\circledR} 3 . x$ was applied for the fusion of CT/MRI and irradiation treatment plans. Overlay images were generated to determine the exact position of the suspected metastases in relation to the prior catheter location.

As a novel approach, we assessed both, extrahepatic and intrahepatic seeding. An example of an image fusion data set is provided in Figure 1. 


\section{Statistical analysis}

We collected technical data of the irradiation plan and documented possible risk factors such as patient demographics, histological grading, and imaging features.

The statistical analysis of the data was conducted by using the statistical software SPSS ${ }$ and $S A S \circledast$. Differences between variables were examined using Student's $t$-test for metric variables and Chi-Square test for frequencies. The survival analysis was performed according to Kaplan-Meier method, the statistical significance was determined using log-rank test. The influence of potential risk factors on the occurrence of needle track metastases was calculated using a generalized linear mixed model. All tests were performed two-sided, a $p$-value of $p \leq 0.05$ was considered statistically significant.

\section{Results}

\section{Patient and treatment characteristics}

In our cohort of 100 patients, a total of 233 HCC lesions were treated. In 62 patients, histological reports were available with $38.7 \%(n=24)$ being well differentiated, $54.8 \%(n=34)$ being moderately differentiated, and $6.5 \%(n=4)$ being poorly differentiated tumors. Pseudo-capsular HCC were present in 18 out of 233 lesions $(7.7 \%) .22$ patients $(22 \%)$ received concomitant therapy with sorafenib.

In all patients, thermal ablation was technically not favorable related to either tumor size (exceeding $3 \mathrm{~cm}$ ) or tumor location (proximity to liver hilum or adjacent gastrointestinal structures) of at least one lesion.

The median follow-up was 15.7 months (range, 6-70.2 months). Within the observation period, 67 patients developed a tumor progression with a median progression-free survival of 7.0 months. Median overall survival of all patients was 20.0 months. A summary of patient and treatment characteristics is presented in Table 1.

\section{Catheter-based analysis}

A total number of 588 catheters were placed within 100 patients. The mean insertion length of a single catheter was $12.7 \pm 31.2 \mathrm{~cm}$ (range, $5.7-25.4 \mathrm{~cm}$ ). Four catheters were too remote in relation to the target lesion and were not used for irradiation $(0.7 \%)$. However, these lesions were treated in the same session with more precisely placed catheters. A total of nine needle track metastases were identified yielding an incidence of $1.5 \%$ per catheter placement. Seven out of nine seeding metastases were located within the liver (catheter-based risk for intrahepatic seeding, 1.2\%). Two metastases occurred within the peritoneal cavity in the location of a former catheter path (catheter-based risk for extrahepatic seeding, $0.3 \%$ ).

\section{Lesion-based analysis}

A total of $233 \mathrm{HCC}$ lesions were treated. The mean diameter of HCC nodule was $3.3 \pm 2.6 \mathrm{~cm}$ (range, $1.0-16.6 \mathrm{~cm}$ ) requiring a mean number of 2.6 catheters per lesion to ensure a sufficient dose application. The mean applied radiation dose at the tumor rim was $16.5 \pm 11.6 \mathrm{~Gy}$.
The mean sum of in-body catheter length per lesion was $32.1 \pm 37.4 \mathrm{~cm}$ (range, 5.9-247.0 cm). Over-penetration of HCC nodule was found in 53 cases $(9.0 \%)$, with a mean over-penetration length of $1.2 \mathrm{~cm}$ (range, 0.1-2.8 cm). The cumulative frequency of needle track metastases per treated tumor was 3.9\% (intrahepatic location, $3.0 \%$; extrahepatic location, $0.9 \%$ ).

\section{Patients-based analysis}

In our cohort of 100 patients, an average number of 5.9 catheters were placed per patient leading to a mean total in-body catheter length of $74.8 \pm 57.3 \mathrm{~cm}$ (range, 8.6$288.8 \mathrm{~cm}$ ). Imaging analysis revealed needle track metastases in 9 patients. The mean time of occurrence of needle track seeding was 5.5 months (range, 4.8-6.2 months).

\section{Risk assessment}

Needle track seeding occurred in a median time interval of 5.5 months (range, 4.8-6.2 months). No increased risk was found for the tumor grading, age, and sex.

In a catheter-based analysis, we found more frequent seeding in smaller HCC lesions $(p=0.09)$. Liver cirrhosis and underlying etiology had no significant influence on the development of needle track seeding; the same was seen for pseudo-capsular HCC. Treatment-related parameters such as catheter insertion lengths, over-penetration of the lesion (i.e., with the possibility of dislocating tumor cells beyond the lesions into the liver parenchyma), and applied dose as well as concomitant treatment with sorafenib, demonstrated no significant influence.

Of note, 8 out of 9 seeding metastases were successfully treated by further HDR-BT directly after their occurrence. In one case, needle track metastasis occurred parallel to systemic progression at other sites and needed systemic therapy with sorafenib.

Median overall survival was 25.0 months in patients with needle track vs. 20.0 months in patients without $(p=0.86, \log$-rank test). The overall results of the risk factor analysis are included in Table 1.

\section{Discussion}

We were able to calculate the risk for tumor seeding after local ablation of HCC by catheter-based radiotherapy for both intrahepatic and extrahepatic locations, with an analysis of catheter-, lesion-, and patient adjusted frequencies. No track irradiation had been used in these patients.

\section{Needle track seeding in local ablation}

Needle track seeding in HCC is known to occur after diagnostic biopsies and local ablative procedures such as radiofrequency or microwave ablation. Stigliano et al. reported a meta-analysis of diagnostic and therapeutic interventions in 2007, with an overall frequency of $1.27 \%$ after liver biopsy and/or local ablation with extrahepatic needle track metastases [11].

Initial reports of seeding in up to $12.5 \%$ of patients after RFA illustrated the demand of track ablation tech- 
niques [18]. Similarly, recent reports after RFA and MWA using track ablation depict low seeding rate of $0.61 \%$ to $1.6 \%[13,19]$. However, all these studies have focused on extrahepatic seeding only; intrahepatic seeding was not evaluated to differentiate tumors seeding from de novo HCC due to technical limitations.

Our recent study identified a cumulative (extrahepatic and intrahepatic) catheter-based risk for seeding of $1.5 \%$ (without track ablation technique), which is comparable to reported seeding risk after thermal ablation using track ablation or even lower, considering that literature focusses on extrahepatic seeding only.

Due to the need of multiple catheters in larger HCC lesions (mean lesion diameter in our cohort: $3.3 \pm 2.6 \mathrm{~cm}$; range, $1.0-16.6 \mathrm{~cm}$ ), the cumulative lesion-based seeding risk is as high as $3.9 \%$. Theoretically, the seeding risk is still comparable to thermal ablation techniques, considering the need for multiple probes/multiple positions and in RFA or MWA ablation for the treatment of larger tumors.

\section{Extrahepatic seeding}

As stated above, our data indicates that the risk for extrahepatic seeding $(0.2 \%$ per catheter) after HDR brachytherapy is comparable or even lower than after thermal-based ablative techniques (0.61-1.6\%). Furthermore, our data supports findings previously published by Denecke et al. who utilized HDR brachytherapy in the pre-transplant setting and found no extrahepatic recurrence due to seeding in their smaller group of patients undergoing subsequent liver transplantation [9]. In fact, only a minority of seeding metastases occurred outside the liver in our cohort ( $0.2 \%$ per catheter). Assuming a tumor size and tumor number within transplant criteria for HCC, the lesion-based and patient-based risk should be equal or only slightly increased in those patients as compared to the catheter-based risk supporting the findings of Denecke et al. Both, the work of Denecke et al. and our results support the use of HDR brachytherapy as bridging for transplant, at least in tumors with an unfavorable location for RFA or TACE.

In case of larger or multilocular HCC outside transplant criteria, multiple catheter placements in HDR brachytherapy are usually necessary, resulting in a higher seeding rate (e.g., $0.9 \%$ in lesion-based analysis). Theoretically, several needle positions would have been required for a complex thermal ablation in those patients. Thus, the cumulative risk (i.e., lesion- and catheter-based risk) for track seeding seen in our study can be assumed to be comparable to a cumulative risk resulting from multiple ablation positions in RFA/MWA [11,20,21].

\section{Intrahepatic seeding}

Unfortunately, many studies still neglect the possibility of intrahepatic seeding, probably as the differentiation between intrahepatic seeding metastases and tumor progression is difficult [12]. We applied a novel approach of image fusion for the identification of intrahepatic seeding, leading to the confident identification of lesions, which most likely derive from track seeding. All these lesions are omitted by the extrahepatic definition of seeding in most studies. In fact, intrahepatic needle track metastases were more frequent as compared to extrahepatic needle track metastases with a catheter-based risk of $1.3 \%$. This is easily explainable, since the penetration depth within the liver parenchyma is usually longer than the thickness of the abdominal wall.

The higher rate of intrahepatic seeding as compared to extrahepatic seeding in our analysis, along with the focus on extrahepatic-only seedings in literature, suggests that seeding (intrahepatic plus extrahepatic) after thermal ablation or biopsy might be more frequent, but data to further elucidate that matter is not available. However, this might pose a clinical impact for treatment decision making and should be a subject for further investigation. Furthermore, techniques to decrease the seeding rate after HDR brachytherapy were not applied in the study population. As a consequence of our analysis, we established a procedure similar to needle track ablation by radiation of the path of the catheter during the withdrawal of the Iridium 192 source, with a mean dose of $10 \mathrm{~Gy}$ in up to 2-3 mm depth. Taking RFA and MWA as an example, the introduction of track ablation techniques resulted in drastically lower rate of (extrahepatic) seeding (from 12.5\% to $0.61-1.6 \%$; see above), indicating a significant influence of track ablations techniques to control tumor seeding in local ablations of HCC. We believe that this applies also to HDR brachytherapy.

Beside this assumed influencing factor on track seeding, none of the evaluated variables within the study revealed a significant influence on the rate of track seeding on a catheter-, lesion-, and patient-based analysis, as these were in particular sex, age, etiology of liver disease, grading of the HCC, evidence of a tumor pseudo-capsular, size of the targeted lesion, in situ catheter length, over-penetration of the targeted lesion, ablation dose, and concomitant systemic treatment. Only the size of targeted lesion showed a tendency to significantly influence track seeding (in the patient-based analysis, $p=0.09$ ), with a higher rate of track seeding in smaller lesions. It can be hypothesized that more manipulations (i.e., needle passes to enable a sufficient catheter placement) are needed in smaller lesions. Quantitative or semi-quantitative information on needle manipulations during the intervention were not available in this study making further clarification of this hypothesis impossible. However, since this finding was only evident on a patient-based analysis but not on a lesion- or catheter-based analysis, stochastic effects are the most probable cause.

\section{Limitations}

The limitations of this analysis are those inherent to a retrospective analysis. Although study format was retrospective, the data (clinical data, treatment-related data) were obtained from a prospectively managed database, in which all patients who undergo a local or loco-regional treatment at our department are electronically filed using standardized reporting forms for treatment and follow-up visits. Additionally, treated patient undergo 
a standardized follow-up including imaging (at our institution) every three months, which diminishes a possible bias derived from inconsistent image follow-up intervals and inconsistent imaging protocols. However, a patient selection bias cannot be ruled out.

As pointed out in the section above, we were not able to evaluate systematically the incidence of needle manipulations during the interventions, which could have an influence on the risk of track seeding, since the number of possible tumors passes increases. However, misplacements of the needle during the intervention usually occur outside the tumor (i.e., in the liver parenchyma without risk for seeding), a believed position within the tumor (although position might not be perfect for treatment) entail the exchange to the brachytherapy catheter by standard operating procedure in order to prevent seeding. Thus, we believe that the possible influence of needle manipulations during the interventions is too small to neglect.

Finally, the differentiation between iatrogenic track seeding and de novo HCC is still a challenge that might influence the analysis. Since all possible track seeding metastases were verified in their origin by precise image registration of the follow-up imaging with the final imaging after placement of the brachytherapy catheters, we can rule out an underestimation of the frequency of treatment-associated track seeding. Only a risk for an overestimation of the frequency of new metastases related to the previously performed local ablation is possible but is regarded as acceptable from a clinical and scientific perspective.

\section{Conclusions}

The technique of percutaneous catheter placement for HDR brachytherapy in HCC is generally not associated with an elevated risk of needle track metastases as compared to biopsy or RFA/MWA, especially in extrahepatic seeding. In fact, data indicates a lower risk for track metastases after HDR brachytherapy as compared to biopsy and thermal-based ablation techniques, although HDR brachytherapy was conducted without a track ablation technique in this study.

To further reduce the risk of seeding along the catheter path, track irradiation in HDR brachytherapy should be implemented in daily practice.

\section{Disclosure}

Authors report no conflict of interest.

\section{References}

1. Schütte K, Kipper M, Kahl S et al. Clinical characteristics and time trends in etiology of hepatocellular cancer in Germany. Digestion 2013; 87: 147-159.

2. Han K, Kim JH. Transarterial chemoembolization in hepatocellular carcinoma treatment: Barcelona clinic liver cancer staging system. World J Gastroenter 2015; 21: 10327-10335.

3. Feng K, Ma KS. Value of radiofrequency ablation in the treatment of hepatocellular. World J Gastroenterol 2014; 20: 5987-5998.

4. Tanaka M, Ando E, Simose S et al. Radiofrequency ablation combined with transarterial chemoembolization for intermediate hepatocellular carcinoma. Hepatol Res 2014; 44: 194-200.
5. Terzi E, Piscaglia F, Forlani L et al. TACE performed in patients with a single nodule of hepatocellular carcinoma. BMC Cancer 2014; 14: 601.

6. Yang W, Yan K, Wu GX et al. Radiofrequency ablation of hepatocellular carcinoma in difficult locations: Strategies and long-term outcomes. World J Gastroenterol 2015; 21: 1554-1566.

7. Mohnike K, Wieners G, Schwartz F et al. Computed tomography-guided high-dose-rate brachytherapy in hepatocellular carcinoma: safety, efficacy, and effect on survival. Int J Radiat Oncol Biol Phys 2010; 78: 172-179.

8. Collettini F, Singh A, Schnapauff D et al. Computed-tomography-guided high-dose-rate brachytherapy (CT-HDRBT) ablation of metastases adjacent to the liver hilum. Eur J Radiol 2013; 82: e509-514.

9. Denecke T, Stelter L, Schnapauff D et al. CT-guided Interstitial Brachytherapy of Hepatocellular Carcinoma before Liver Transplantation: An Equivalent Alternative to Transarterial Chemoembolization? Eur Radiol 2015; 25: 2608-2616.

10. Cabibbo G, Craxi A. Needle track seeding following percutaneous procedures for hepatocellular carcinoma. World J Hepatol 2009; 1: 62-66.

11. Stigliano R, Marelli L, Yu D et al. Seeding following percutaneous diagnostic and therapeutic approaches for hepatocellular carcinoma. What is the risk and the outcome? Seeding risk for percutaneous approach of HCC. Cancer Treat Rev 2007; 33: 437-447.

12. Francica G. Needle track seeding after radiofrequency ablation for hepatocellular carcinoma: prevalence, impact, and management challenge. J Hepatocell Carcinoma 2017; 4: 23-27.

13. Yu J, Liang $\mathrm{P}, \mathrm{Yu} \mathrm{XL}$ et al. Needle track seeding after percutaneous microwave ablation of malignant liver tumors under ultrasound guidance: analysis of 14-year experience with 1,462 patients at a single center. Eur J Radiol 2012; 81: 2495-2499.

14. Collettini F, Schnapauff D, Poellinger A et al. Hepatocellular carcinoma: computed-tomography-guided high-dose-rate brachytherapy (CT-HDRBT) ablation of large $(5-7 \mathrm{~cm})$ and very large (> $7 \mathrm{~cm}$ ) tumours. Eur Radiol 2012; 22: 1101-1109.

15. Bruix J, Sherman M, Llovet JM et al. Clinical management of hepatocellular carcinoma. Conclusions of the Barcelona-2000 EASL conference. European Association for the Study of the Liver. J Hepatol 2001; 35: 421-430.

16. European Association for The Study Of The L, European Organization For R, Treatment Of C. EASL-EORTC clinical practice guidelines: management of hepatocellular carcinoma. J Hepatol 2012; 56: 908-943.

17. Ricke J, Wust P. Computed tomography-guided brachytherapy for liver cancer. Semin Radiat Oncol 2011; 21: 287-293.

18. Llovet JM, Vilana R, Bru C et al. Increased risk of tumor seeding after percutaneous radiofrequency ablation for single hepatocellular carcinoma. Hepatology 2001; 33: 1124-1129.

19. Zhong-Yi Z, Wei Y, Kun Y et al. Needle track seeding after percutaneous radiofrequency ablation of hepatocellular carcinoma: 14-year experience at a single center. Int J Hyperthermia 2017; 33: 1-20.

20. Shirai K, Tamai H, Shingaki $\mathrm{N}$ et al. Clinical features and risk factors of extrahepatic seeding after percutaneous radiofrequency ablation for hepatocellular carcinoma. Hepatol Res 2011; 41: 738-745.

21. Silva MA, Hegab B, Hyde C et al. Needle track seeding following biopsy of liver lesions in the diagnosis of hepatocellular cancer: a systematic review and meta-analysis. Gut 2008; 57: 1592-1596. 